\title{
Characterisation of the Layer Pressing Strategy for Concrete 3D Printing
}

\author{
Paul Carneau ${ }^{1}$, Romain Mesnil ${ }^{1}$, Nicolas Ducoulombier ${ }^{1}$, Nicolas Roussel $^{1}$, and \\ Olivier Baverel ${ }^{1}$ \\ Laboratoire Navier, UMR 8205, Ecole des Ponts, CNRS, UGE, Champs-sur-Marne, \\ France
}

\begin{abstract}
. 3d printing of cementitious material by pressing layers during the extrusion is a strategy that requires a rather low initial yield stress so the material can deform without cracking. It allows to perfectly control the height of the layer and gives freedom in the orientation of the printing head and of the layer allowing for a wider range of printable geometry than the classic so-called "infinite brick" extrusion. This strategy has however some drawbacks as pressing the material on the previous layers may lead to a deformation of the sub-layers and even failure of the structure. In this work; we make a first step into understanding forces involved in such a process and measure their dependency on material fresh properties and printing parameters.
\end{abstract}

Keywords: $3 \mathrm{~d}$ concrete printing $\cdot$ yield stress $\cdot$ layer pressing $\cdot$ printing parameters · geometry control

\section{Introduction}

The development of $3 \mathrm{~d}$ printing of cementitious materials has led to multiple solutions or strategies for extruding layers, each one opening new paths for research and development [1] . Fig. 2 illustrates in an asymptotic way these strategies at the level of the nozzle. The infinite brick extrusion consists in extruding unsheared material through a nozzle that imposes its shape to the layer. The simplicity of this process is balanced by the limitations in terms of achievable global shapes (i.e. layers can only be stacked vertically) and control over the layer geometry (i.e. in-line monitoring of the geometry may prove necessary due to error accumulation [2]). The free flow deposition lacks any control over the layer geometry, only the volume of extruded material is known and the final shape results from a complex competition between gravity and thixotropy [3]. An example of application of this strategy is developed in [4].

The third strategy consists in pressing the layers during the extrusion so that the height of the layer is always imposed by the relative position of the nozzle. The material is then free to deform between the substrate (or the previous layer) and the nozzle giving to this process the freedom to allow for a potential inclination of the printing head and, in turn, of the printed layer [5] (note that curved layer can be printed without inclining the extrusion head [6], but the 
generation of the toolpath becomes difficult). It moreover allows for variations in the layer cross section during a print by playing on one of the printing parameters involved, namely the printing speed $V_{r}$, the extrusion speed $V_{e}$ or the extrusion height $H$.

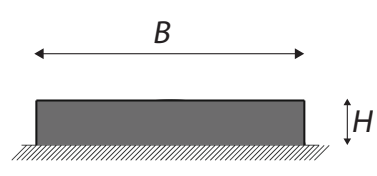

(a) Infinite brick extrusion

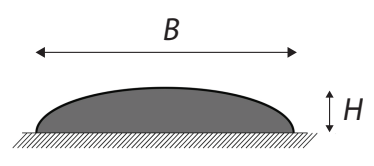

(b) Free flow deposition

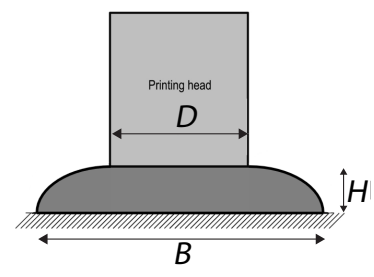

(c) Layer pressing strategy

Fig. 2: Different extrusion strategies for $3 \mathrm{~d}$ printing at the nozzle

The drawbacks with the such a printing strategy is that while pressing the layers, an additional force is applied on the previously printed layers. If the induced stress exceeds the yield stress of the material, this may lead to periodic deformations shown in Fig. 3, left or even to a global failure of the object (Fig. 3 , right).

Within this frame, the goal of the present paper is to assess the magnitude of this additional force occurring locally during the extrusion, and inform the design and fabrication processes with it.
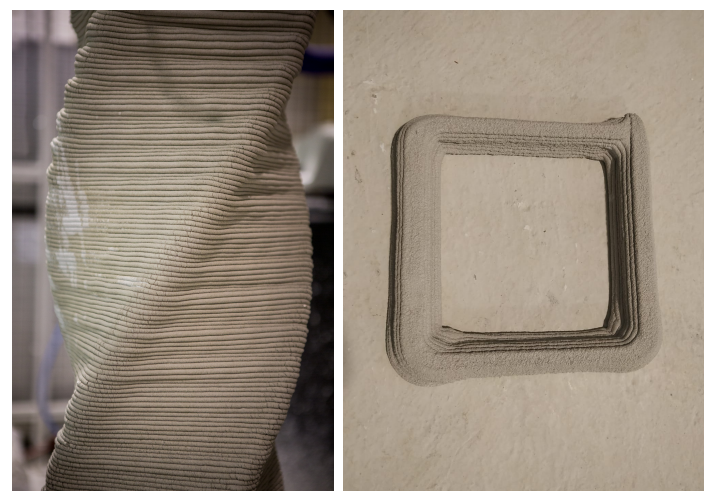

Fig. 3: left: wave pattern on a column appearing during printing (photo credits: Stefano Borghi), right: plastic collapse on a 10 layers piece due to the extra force applied on substrate during the extrusion (photo credits: Paul Carneau). 


\subsection{Method Statement}

In a first part, we focus on the geometric characterisation of a layer which is pressed by the nozzle on the substrate. Our results illustrates how the layer's section is different from the nozzle geometry due to the material deformation through the extrusion/deposition process. In a second part we develop an experimental protocol allowing for the measurement of the additional force induced by the pressing of the extruded layers. Finally, we discuss the results obtained by the experiment in regards to layer geometry and material properties.

\section{Geometric characterisation of the layer's section}

The layer pressing strategy relies on kinematic parameters, geometrical parameters and material parameters, all of which are listed in table 1. In the following, we consider a Bingham fluid, with yield stress $\tau_{c}$ and plastic viscosity $\nu$. The proposed model could however be extended to more complex models for yield stress fluids.

The process of pressing layers is characterised by a fluid extruded at a velocity $V_{e}$ through a nozzle of diameter $D$ and height $H$, by a robot moving at a speed $V_{r}$. The result is a layer of width $B$ (independent of the nozzle diameter $D$ ) and whose height $H$ is directly given by the height of the nozzle.

Table 1: $3 \mathrm{~d}$ printing process parameters: bold values can be independently controlled.

\begin{tabular}{|l|c|c|c|}
\hline Parameter & Symbol & Unit & Category \\
\hline Robot speed & $V_{r}$ & $\mathrm{~m} . \mathrm{s}^{-1}$ & kinematic \\
Extrusion speed & $V_{e}$ & ${\mathrm{~m} . \mathrm{s}^{-1}}$ & \\
\hline Height of layer & $H$ & $\mathrm{~m}$ & \\
Width of layer & $B$ & $\mathrm{~m}$ & Geometric \\
Nozzle diameter & $D$ & $\mathrm{~m}$ & \\
\hline Yield stress & $\tau_{c}$ & $\mathrm{~Pa}$ & \multirow{2}{*}{ Material } \\
Dynamic viscosity & $\mu$ & $\mathrm{Pa} . \mathrm{s}$ & \\
\hline
\end{tabular}

This section studies the kinematic and geometric parameters of the printing process. The goal is to determine the parameter range allowing to control the layer's geometry for the experimental validation, which is presented in the next section.

\subsection{Lace stretching}

If we consider that the nozzle diameter $D$ stays unchanged through the printing process, we can introduce the nozzle section as $d S=\pi D^{2} / 4$. We call $d S^{\prime}$ the cross section of a layer, which can vary continuously during a print by changing 
any of the control parameters (robot speed $V_{r}$, flow rate $V_{e}$ or height $H$ ). The ratio $d S / d S^{\prime}$ between initial and final material cross section provides information on the imposed strain and therefore on the longitudinal stresses in the layer. Indeed, if the final cross section is larger than the nozzle cross section, the layer is compressed. If this ratio is smaller than one, the material undergoes a striction and the layer is extruded with a longitudinal tensile stress which can lead to cracks or discontinuity as shown in Fig. 4. In [7], a study of printing parameters impacting the cracking is conducted in order to voluntarily create weak parts in an object that can easily be removed afterwards.

The conservation of mass implies that $V_{e} d S=V_{r} d S^{\prime}$. The above section ratio can therefore be rewritten as a ratio between velocities, which are the printing parameters that are the relevant parameters used in practice. In the following, we will target a ratio $V_{r} / V_{e}$ around 1 to avoid the generation of any longitudinal stresses and isolate the vertical consequences of pressing layers.

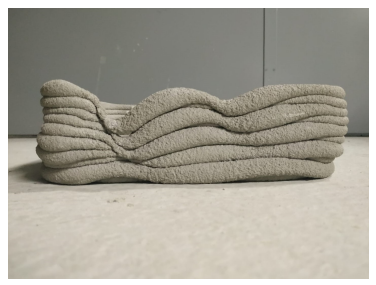

Fig. 4: Longitudinal tearing in the extruded material creating discontinuous layers (photo credits: Paul Carneau)

\subsection{Shape of the layer profile after pressing}

The first objective is to estimate the shape of the deformed layer after pressing. Indeed, the width $B$ cannot directly be controlled by the printing operator, but rather indirectly imposed by controlling the other printing parameters. The layer pressing strategy offers the freedom to modify parameters during the printing process to modify the geometry of the layer that can fit design requirements (like for example having thicker wall at the base than at the top). The counterpart is the necessity for the user to understand and calibrate the indirect impact of each control parameter on the resulting layer cross section.

Using the fact that $d S=\pi D^{2} / 4$, and introducing a shape parameter $\beta$ so that $d S^{\prime}=\beta B H$, we have :

$$
\frac{\pi D^{2}}{4 B H}=\beta \frac{V_{r}}{V_{e}}
$$

$\beta$ describes the discrepancy between the real section of the layer and a ideal rectangular section of width $B$ and height $H$ as illustrated in Fig. 5. We have thus for example $\beta=1$ for a rectangular section and $\beta=\pi / 4$ for an elliptical profile. 


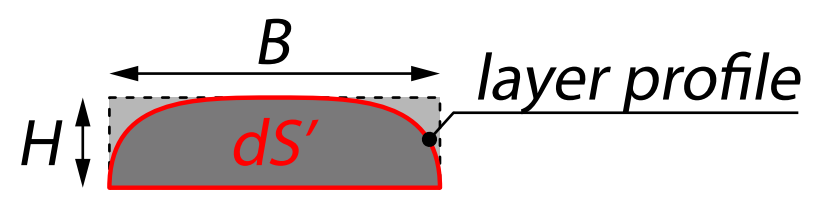

Fig. 5: Real layer profile compared to an ideal rectangular section of area $H B$

\subsection{Kinematic conditions for pressing layers}

As mentioned above, the main interest in pressing the layers is the capacity to control the height of the layer and, in case of deformation of the layers below, to recover from such discrepancies by ensuring that the height of the layer equals the distance between the nozzle and the substrate.

To achieve this, the first condition is related to the material properties as the layer has to withstand its own weight [8], [9]:

$$
\frac{\rho g H}{\sqrt{3}} \leq \tau_{0}
$$

The second condition is purely kinematic and it has previously been identified for viscoelastic material [10]. Let us consider a part of a layer of length $D$ that has just been extruded at a velocity $V_{r}$. The time to extrude this volume of material is obviously $t_{c}=\frac{D}{V_{r}}$. This characteristic time is the duration the robot spends above a specific section of the layer.

Before being extruded, this volume of material was a cylinder of diameter $D$ and height $L_{c}=V_{e} \times t_{c}=\frac{D V_{e}}{V_{r}}$ inside the extrusion head. A straightforward condition for pressing the layer, illustrated in Fig. 6 can be set as the height of the cylinder inside the nozzle $L_{c}$ must be larger than the distance $H$ between the nozzle and the sub-layers. This condition reads:

$$
H<\frac{D V_{e}}{V_{r}}
$$

\section{$3 \quad$ Experimental study of vertical loading}

The above considerations can only be valid if a pressure force due to the extrusion process and independent from the material weight actually exist. The following experiment aims at measuring for the first time this additional force and assess its amplitude and comparing it to the layers weight.

In the experimental set-up, the robot extrudes layers directly onto a scale which measures thus the cumulative weight of the laces along with the force $F$ due to pressing. Each series of test is composed of 5 laces, the robot following the toolpath shown in Fig. 7. The plate surrounding the scale allows to keep the pressure in the extrusion head even when not above the scale in order to eliminate variation in the measures coming from a refilling of the tube at the 


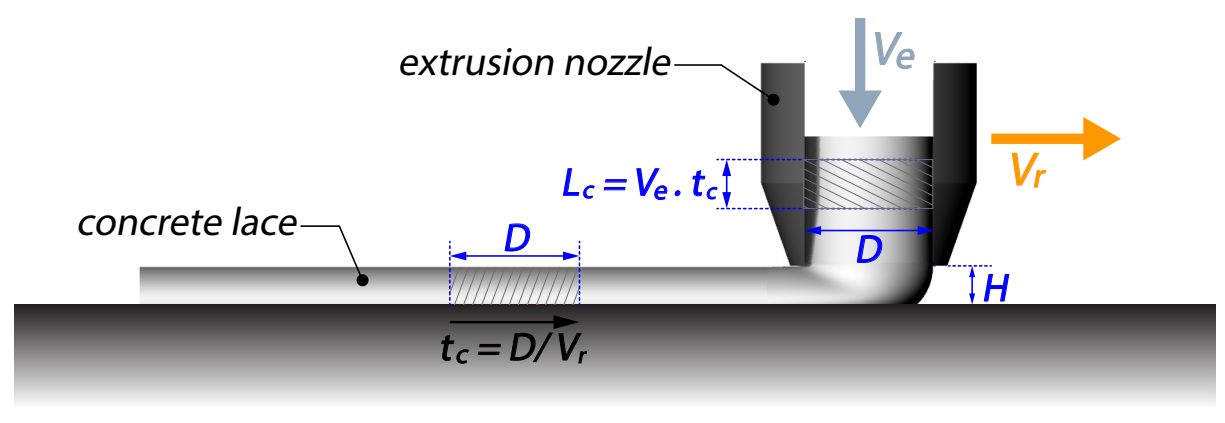

Fig. 6: The layer is pressed if the height $L_{c}$ is larger than the final height $H$.
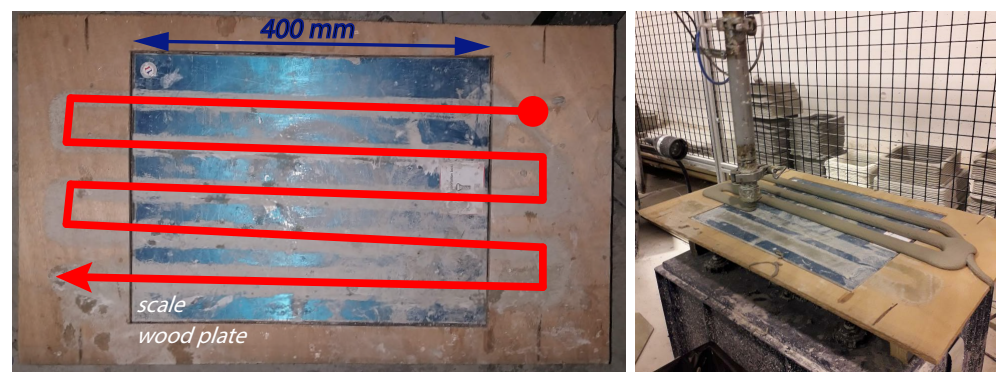

Fig. 7: left: Experimental apparatus with a scale and surrounded plate, and toolpath to measure the force applied during the extrusion, right: On-going printing of the layers on the scale (photo credits: Romain Mesnil).

start of each new lace. The layers are printed in decreasing height order (from larger $H$ to smaller).

The composition of the material used for this experiment is detailed in table 2 for information. Indeed the equations introduced in this article involving stresses are valid for any yield stress material and the geometric and kinematic conditions are valid for any incompressible fluid.

The yield stress of the material is determined before each series using the protocol described in [11]. Table 3 below shows the parameters used for each series of tests.

The printing velocity and flow rate are imposed by the manipulator directly to the robot and the dosing pump equipped on the extrusion head. Although the robot accuracy is reliable, it can be verified by dividing the toolpath length by the deposition time measured on Fig. 8 .

The width of the layers $B$ is measured physically just after extrusion. Fig. 8 shows the weight measured by the scale as a function of time for each of the three series of tests. On each curve, the five different paths can be identified, the overall 
Table 2: Premix composition

\begin{tabular}{|l|c|}
\hline Components & Mass ratio \\
\hline Portland cement CEM I & $30-40 \%$ \\
\hline Crystalline Silica & $40-50 \%$ \\
\hline Silica fume & $10 \%$ \\
\hline Limestone filler & $10 \%$ \\
\hline water/powder ratio & 0.1 \\
\hline
\end{tabular}

slope giving the exact flow rate achieved during the experiment. This exact flow rate can differ from the value imposed to the pump and is thus preferred for the rest of the calculations.

The additional force $F$ to measure can be identified from the graph as the jump between the horizontal plateau and the linear part of the curve for each layer. It can be noted at this stage, that this jump increases as the layer height $H$ decreases.

To measure this force precisely, we subtract the self-weight of the material to the total measurement. This way, we ensure that $F$ does not include the weight of the material.

Table 3: Experimental parameters and results

\begin{tabular}{|c|c|c|c|c|c|c|c|c|c|c|c|c|c|}
\hline \multicolumn{2}{|l|}{ Serie } & \multicolumn{4}{|c|}{ A } & \multicolumn{4}{|c|}{$\bar{B}$} & \multicolumn{4}{|c|}{$\mathbf{C}$} \\
\hline parameter & unit & 1 & 2 & 3 & \begin{tabular}{|l|l|}
4 & 5
\end{tabular} & 1 & 2 & 3 & $4:$ & 1 & 2 & 3 & \begin{tabular}{|l|l|}
4 & 5
\end{tabular} \\
\hline density & - & \multicolumn{4}{|c|}{2484} & \multicolumn{4}{|c|}{2512} & \multicolumn{4}{|c|}{2512} \\
\hline yield stress & $P a$ & \multicolumn{4}{|c|}{500} & \multicolumn{4}{|c|}{200} & \multicolumn{4}{|c|}{200} \\
\hline nozzle diameter $D$ & $m m$ & \multicolumn{4}{|c|}{20} & \multicolumn{4}{|c|}{20} & \multicolumn{4}{|c|}{20} \\
\hline velocity $V_{r}$ & $\mathrm{~mm} / \mathrm{s}$ & \multicolumn{4}{|c|}{41.5} & \multicolumn{4}{|c|}{55.4} & \multicolumn{4}{|c|}{41.5} \\
\hline flow rate & $g / s$ & \multicolumn{4}{|c|}{33.7} & \multicolumn{4}{|c|}{40} & \multicolumn{4}{|c|}{18.6} \\
\hline height $H$ & $m m$ & \multicolumn{4}{|c|}{\begin{tabular}{|l|l|l|l|l|}
20 & 15.7 & 12 & 10 & 8 \\
\end{tabular}} & \multicolumn{4}{|c|}{\begin{tabular}{|l|l|l|l|l|}
20 & 15.7 & 12 & 10 & 8 \\
\end{tabular}} & \multicolumn{4}{|c|}{\begin{tabular}{|l|l|l|l|l|}
20 & 15.7 & 12 & 10 & 8 \\
\end{tabular}} \\
\hline
\end{tabular}

\section{Results and discussions}

\subsection{Layer geometry control}

The first result concerns the geometry control of a layer. Fig. 9 gives coefficient $\beta=d S^{\prime} / B H$ as a function of the target height of the layer $H$. For smaller values of $H, \beta$ shows a plateau whose length is linked to the yield stress of the material $\tau_{0}$. Then when the height increases, $\beta$ decreases with a constant slope. This part corresponds to layers printed without any exact control over the geometry. 


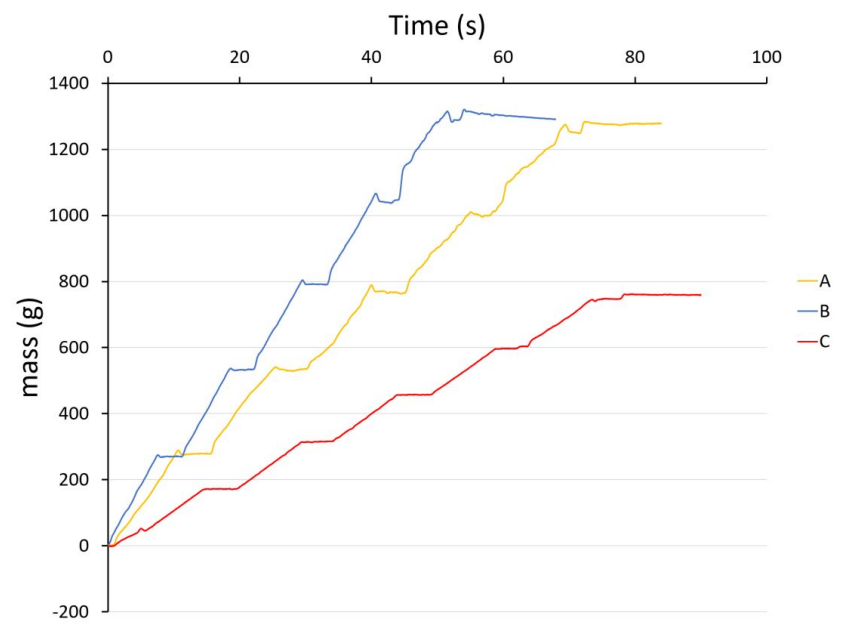

Fig. 8: Mass measured by the scale in function of the time for the three series of test.

The final height of the layers is different from the target height $H$ due to an uncontrolled flow of the material under a combination of its own weight and of the force applied during the extrusion.

In the case where the robot speed and the extrusion velocity are of the same magnitude (series $A$ and $B$ ) the value $\beta=0.8$ provides a good evaluation of the exact layer cross section.

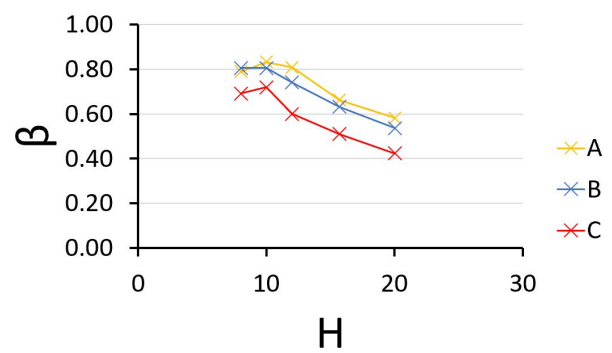

Fig. 9: Coefficient $\beta$ for the different series of experiment. $\beta$ reflects the geometry control of the layer. 


\subsection{Self-weight and applied force}

In this section we compare the stress induced by the self-weight of the layer $w$ and the stress coming from the extrusion and pressing process $\tau_{e}$ only:

$$
\left\{\begin{aligned}
w & =\frac{\rho g H}{\sqrt{3}} \\
\tau_{e} & =\frac{4 F}{\pi D^{2}}
\end{aligned}\right.
$$

The results are presented in Fig. 11 for each three series of experience. As expected, $w$ evolves linearly with $H$ while $\tau_{e}$ seems to be inversely proportional to it. Therefore, as $H$ decreases, the force due to the extrusion becomes preponderant over the material self-weight.

In the case of the infinite brick extrusion strategy, where all stresses comedown to the weight of the material only, Kruger et al. have developed a model [12] to optimise printing parameters. With the layer pressing strategy, such a model shall be completed by accounting for the contribution of the stresses coming from the pressing force and its influence on the failure modes [13].
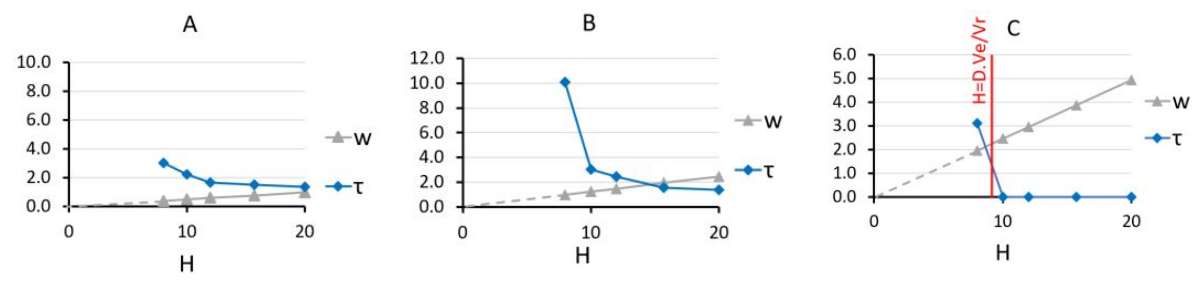

Fig. 11: Comparison of non-dimensional stresses $w *$ and $\tau *$ for the three series of tests

Looking at the results of serie $\mathrm{C}$ in Fig. 11, it appears that no force was measured by the scale other than the one coming from the self-weight. This can be explained by drawing the layer pressing criteria described earlier $H<\frac{D V_{e}}{V_{r}}$ on the graph (see the red line). It separates exactly the laces where an additional force was measured from the laces that were simply deposited. This seems to validate our layer pressing condition as a good first evaluation of the parameters toward a better control of the layer geometry. In addition, by observing that $\frac{D V_{e}}{V_{r}} \propto \frac{Q}{D V_{r}}$, the user can ensure the condition is fulfilled by simply changing the nozzle diameter, without impacting the layer geometry.

Finally, to give a better understanding of the amplitude of the pressing force compare to the weight of the material, we plot in Fig. 12 the ratio between the pressing force and the weight of one layer $\tau_{e} / w$ as a function of the height of the layers. The $\mathrm{Y}$-axis corresponds therefore to the number of layers whose total weight is equivalent to the pressing force acting on the substrate. 


\section{Layers equivalent}

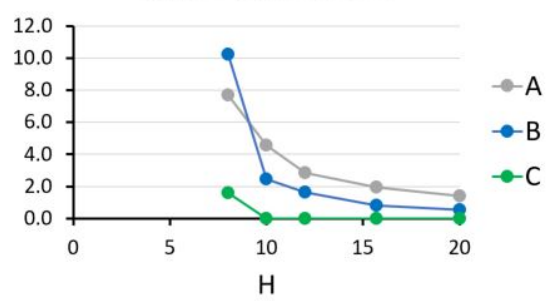

Fig. 12: Pressing force expressed in terms of number of layers of equivalent height $H$.

\section{Conclusion}

The layer pressing strategy for $3 \mathrm{~d}$ printing of cementitious material offers interesting perspectives in terms of achievable geometries and innovative printing strategies. However the additional force induced by the pressing of the extruded material on the sub-layers may lead to a local failure of the sub-layers independently of the weight of the material. The experience described in this paper highlights first the impact of the measured force on the control of the layer section geometry. It then compares it to the self-weight of the material to justify the importance of taking it into account when choosing the printing parameters.

\section{References}

1. R. Buswell, W. R. Leal da Silva, S. Z. Jones, J. Dirrenberge, "3D printing using concrete extrusion: A roadmap for research", in Cement and Concrete Research. October 2018, pp 37-49. 10.1016/j.cemconres.2018.05.006

2. R. Wolfs, F. Bos, E. van Strien, T. Salet, "A real-time measurement and feedback system for 3d concrete printing" in High Tech Concrete: Where Technology and Engineering meet, 2018. 978-3-319-59471-2

3. L. Reiter, T. Wangler, N. Roussel, R. Flatt, "The role of early age structural buildup in digital fabrication with concrete", in Cement and Concrete Research.October 2018, pp 86-95. 10.1016/j.cemconres.2018.05.011

4. R. Duballet, R. Mesnil, N. Ducoulombier, P. Carneau, L. Demont, M. Motamedi, O. Baverel, J.-F. Caron, J. Dirrenberger, "Free deposition printing for space truss structures" in Digital Concrete 2020, (under review), 2020.

5. P. Carneau, R. Mesnil, N. Roussel, O. Baverel, "An exploration of 3d printing design space inspired by masonry" in Proceedings of IASS Symposium 2019, "Form and Force". Barcelona, Octobre 2019. 10.5281/zenodo.3563672

6. S. Lim, R. Buswell, P. Valentine, D. Piker, S. Austin, and X. De Kestelier, "Modelling curved-layered printing paths for fabricating large-scale construction components" in Additive Manufacturing. Volume 12, Part B, October 2016, pp 216-230. 10.1016/j.addma.2016.06.004

7. Y. W. D. Tay, M. Y. Ming, J. Ming, "Effect of printing parameters in 3D concrete printing: Printing region and support structures", in Journal of Materials Processing Technology.September 2019, pp 261-270. 10.1016/j.jmatprotec.2019.04.007 
8. A. Perrot, D, Rangeard, A. Pierre, "Structural built-up of cement-based materials used for 3D-printing extrusion techniques", in Materials and Structures. April 2016,pp 1213-1220. 10.1617/s11527-015-0571-0

9. N. Roussel, "Rheological requirements for printable concretes" in Cement and Concrete Research. May 2018. 10.1016/j.cemconres.2018.04.005

10. H. Yuk, X. Zhao, "A new 3D printing strategy by harnessing deformation, instability, and fracture of viscoelastic inks", in Advanced Materials. February 2018. 10.1002/adma.201704028

11. N. Ducoulombier, P. Carneau, R. Mesnil, J.-F. Caron, N. Roussel, "” The Slug test": Rheology and homogeneity assessment for Robotic Extrusion of Yield Stress Fluid" in Digital Concrete 2020, (under review), 2020.

12. J. Kruger, S. Cho; S. Zeranka, C. Viljoen, G. van Zijl, "3D concrete printing parameter optimisation for high rate digital construction avoiding plastic collapse" in Composites. Part B, Volume 183. 2020. 10.1016/j.compositesb.2019.107660

13. R. Wolfs, F. Bos, T. Salet, "Early age mechanical behaviour of 3D printed concrete: Numerical modelling and experimental testing" in Cement and Concrete Research. Volume 106, February 2018, pp 103-116. 10.1016/j.cemconres.2018.02.001 